\title{
SINTOMAS PSIQUIÁTRICOS ENTRE PACIENTES COM DEMÊNCIA ATENDIDOS EM UM SERVIÇO AMBULATORIAL
}

\author{
OSVALDO P. ALMEIDA*
}

\begin{abstract}
RESUMO - Pacientes portadores de quadros demenciais frequentemente apresentam uma série de sintomas neuropsiquiátricos que incluem distúrbios do humor, delírios, alucinações, sintomas vegetativos e alterações da atividade psicomotora. Este estudo foi desenhado com o objetivo de investigar a prevalência de morbidade psiquiátrica entre os pacientes com o diagnóstico clínico de demência (ICD-10) atendidos na clínica de memória da Santa Casa de São Paulo entre fevereiro de 1997 e maio de 1998. O estado mental e cognitivo dos pacientes foram avaliados com uma versão ampliada do SRQ-20 e o MMSE respectivamente. Quarenta e cinco porcento dos 75 pacientes disponíveis para análise apresentavam escores iguais ou maiores do que 8 no SRQ-20, indicando a presença de morbidade psiquiátrica significativa. Sintomas característicos de depressão foram relatados por $69,3 \%$ dos indivíduos da amostra. Ideação persecutória e alucinações auditivas foram descritas por 20,0\% e $16,0 \%$ dos idosos avaliados. Oito pacientes $(10,7 \%)$ descreveram a presença de ideação suicida - todos apresentavam sintomatologia depressiva. Pacientes com escores no SRQ-20 $\geq 8$ ou com ideação suicida eram significativamente mais jovens. Aqueles que relatavam alucinações auditivas apresentavam escores significativamente mais baixos no MMSE. Não se observou diferença significativa entre os sexos quanto à frequência dos sintomas investigados. O exame do estado mental deve ser parte integrante da avaliação do paciente com demência. A detecção e tratamento desses sintomas pode contribuir para diminuir o sofrimento do paciente e o estresse de seus cuidadores.
\end{abstract}

PALAVRAS-CHAVE: demência, sintomas psiquiátricos, morbidade psiquiátrica, distúrbios do comportamento, prevalência, ansiedade, depressão, delírios, alucinações, suicídio, tentativa de suicídio, SRQ20, MMSE, CID-10.

\section{Psychiatric symptoms amongst outpatients with dementia in São Paulo, Brazil}

ABSTRACT - Subjects with dementia often display an array of neuropsychiatric symptoms that include disorders of mood, delusions, hallucinations, vegetative symptoms and psychomotor abnormalities. The present study was designed to investigate the prevalence of psychiatric morbidity amongst patients with the clinical diagnosis of dementia (ICD-10) assessed at a Memory Clinic in São Paulo-Brazil between February 1997 and May 1998. The mental and cognitive state of patients were assessed with an extended version of the SRQ-20 and the MMSE respectively. Thirty-four (45.3\%) out of a total of 75 subjects scored 8 or more on the SRQ-20, indicating the presence of significant psychiatric morbidity. Depressive symptoms were reported by $69.3 \%$ of patients. Persecutory ideas and auditory hallucinations were observed in $20.0 \%$ and $16.0 \%$ of the sample respectively. Eight subjects $(10.7 \%)$ described suicidal ideation - they all displayed depressive symptoms. Patients with scores on the SRQ- $20 \geq 8$ or who described suicidal ideation were significantly younger than their counterparts. Auditory hallucinations were more frequent amongst subjects with lower MMSE scores. There were no sex differences in the distribution of the psychiatric symptoms under investigation. The assessment of patients with dementia should always include a detailed psychiatric examination, as the detection and treatment of such symptoms may contribute to decrease the stress of patients and the burden on carers.

KEY WORDS: dementia, psychiatric symptoms, psychiatric morbidity, behavioural disorders, prevalence, anxiety, depression, delusion, hallucination, suicide, attempted-suicide, SRQ-20, MMSE, ICD-10

\footnotetext{
*Department of Psychiatry and Behavioural Science, University of Western Australia, Australia. Aceite: 7-agosto-1999.

Dr. Osvaldo P. Almeida - UWA Department of Psychiatry and Behavioural Science, Queen Elisabeth II Medical Centre - Perth, WA 6009, Australia. Fax 6189346 2393. Email: osvalm@cyllene.uwa.edu.au
} 
O diagnóstico de demência baseia-se na presença de deterioração da memória e de outras habilidades intelectuais do indivíduo ${ }^{1}$. O desenvolvimento desses sintomas compromete a capacidade funcional do paciente nas atividades do dia-a-dia e traz limitações importantes para sua vida. No entanto, as dificuldades cognitivas não são, com frequência, a maior fonte de sofrimento para o paciente e seus cuidadores. Distúrbios emocionais e do comportamento podem ser observados em 50-100\% dos pacientes ao longo do curso da doença ${ }^{2}$. Estes incluem alterações do humor (sintomas depressivos, euforia, labilidade emocional), delírios, alucinações, apatia, irritabilidade, desinibição, ansiedade, reações catastróficas, agressividade verbal e física, comportamentos estereotipados, andar incessante, insônia, e alterações do apetite e do comportamento sexual. Hope e col. ${ }^{3}$ observaram, que a evolução das alterações comportamentais em pacientes com doença de Alzheimer (DA) é bastante variável, embora a duração de episódios específicos (por exemplo: alucinações, tristeza) tenda a ser prolongada (vários meses). Esse mesmo grupo demonstrou, em outro estudo ${ }^{4}$, que a presença de tristeza, alucinações e agressividade verbal estavam associadas ao aparecimento de agressividade física após 4 anos de seguimento. Da mesma forma, idéias persecutórias correlacionaram-se de forma significativa com o posterior desenvolvimento de hiperatividade física. Esses achados sugerem que as alterações comportamentais de pacientes com demência tendem a ter duração prolongada e, ao desaparecerem, apenas cedem espaço para a manifestação de novos sintomas psiquiátricos. São justamente sintomas como depressão, agressividade, distúrbios do sono, delírios e alucinações que mais causam estresse aos cuidadores de pacientes com demência ${ }^{5-7}$. Assim sendo, é importante que a morbidade psiquiátrica de pacientes com demência seja sempre avaliada e, quando possível, devidamente tratada.

Este estudo teve como objetivo investigar a presença de morbidade psiquiátrica entre os pacientes com diagnóstico de demência avaliados na Clínica de Memória da Unidade de Idosos do Departamento de Saúde Mental da Santa Casa de São Paulo.

\section{MÉTODO}

Os pacientes para este estudo foram recrutados no ambulatório da Unidade de Idosos (UNID) do Departamento de Saúde Mental da Santa Casa de São Paulo entre 25 de fevereiro de 1997 e 4 de maio de 1998. A UNID atende a pacientes com idade igual ou superior a 60 anos que procuram o serviço de forma espontânea ou através de encaminhamento de outros profissionais de saúde. A estruturação das consultas médicas desses pacientes foi descrita de forma detalhada em outra publicação ${ }^{8}$. Resumidamente, todos os pacientes são avaliados com o Mini-Exame do Estado Mental - MMSE ${ }^{9}$ e SRQ-20 (Self Reporting Questionnaire) ${ }^{10,11}$ para a investigação do estado mental atual. O SRQ-20 é instrumento que foi desenhado pela Organização Mundial de Saúde para a detecção de morbidade psiquiátrica na população geral. As 20 questões que compõem a escala têm duas possibilidades de resposta ( $\operatorname{sim} /$ não) e foram desenhadas para abordar sintomas emocionais e físicos associados a quadros psiquiátricos (ex: cefaléias frequentes, queixas de insônia e de alterações de apetite, piora da concentração, nervosismo, cansaço, queixas estomacais, diminuição do interesse pelas atividades rotineiras, pensamentos suicidas, sentimentos de tristeza e de desesperança). Morbidade psiquiátrica significativa está associada à presença de 8 ou mais desses sintomas ${ }^{11,12}$. Três questões suplementares foram incluídas no questionário com o objetivo de avaliar a presença de idéias paranóides ('Sente que alguém quer lhe fazer mal?') e de grandiosidade ('Você é alguém mais importante do que a maioria das pessoas pensa?'), bem como de alucinações auditivas ('Ouve vozes que não sabe de onde vêm, ou que outras pessoas não podem ouvir?'). Além do SRQ-20, uma entrevista aberta foi utilizada para a coleta de informações clínicas suplementares que possibilitassem a realização de um diagnóstico sindrômico de acordo com as diretrizes da CID-10¹.

Um total de 351 casos novos foram atendidos nesse período. Cinquenta e três pacientes $(15,1 \%)$ foram excluídos da análise por não apresentarem registro confiável dos dados referentes ao diagnóstico clínico. Dos 298 pacientes elegíveis, 90 (30,2\%) receberam o diagnóstico clínico de demência. O perfil sintomatológico desses indivíduos foi avaliado de acordo com o SRQ-20 e questões suplementares.

Análise dos dados - Os dados foram analisados com o pacote estatístico 'Stata 5,0'. Análise de tabelas de contingência foi utilizada para a investigação de variáveis categoriais, com o resultado estatístico sendo distribuído como qui-quadrado de Pearson $\left(\chi^{2}\right)$. Teste $t$ de Student foi utilizado para a comparação das médias entre dois grupos. O odds ratio foi calculado para estimar o risco relativo associado a algumas variáveis (por exemplo: sexo). Intervalos de confiança de $95 \%$ foram estimados para as médias $(\mathrm{CI})$, diferença entre as médias $\left(\mathrm{CI}_{\mathrm{d}}\right)$, e para o odds ratio $\left(\mathrm{CI}_{\mathrm{OR}}\right)$. 


\section{RESULTADOS}

Quinze (16,7\%) dos 90 pacientes que receberam o diagnóstico clínico de demência precisaram ser excluídos das análises devido à impossibilidade de obter informações seguras quanto ao estado mental. Os resultados aqui relatados referem-se, portanto, a uma amostra de 75 idosos atendidos nos ambulatórios da Unidade de Idosos do Departamento de Saúde Mental da Santa Casa de São Paulo entre 25 de fevereiro de 1997 e 4 de maio de 1998. A idade média dos pacientes foi 73,01 (CI=71,07 a 75,12$)$. Quarenta e cinco eram mulheres $(60,0 \%)$ e $49,3 \%$ eram casados. Vinte e oito $(37,3 \%)$ pacientes relataram nunca haver frequentado a escola. A prevalência de sintomas psiquiátricos, conforme avaliação feita pelo SRQ-20 e questões suplementares, encontra-se resumida na Tabela 1.

Tabela 1. Frequência de respostas afirmativas no SRQ-20 e questões suplementares entre pacientes com diagnóstico clínico de demência

Questão

1. Tem dores de cabeça frequentes?

2. Tem falta de apetite?

3. Dorme mal?

4. Assusta-se com facilidade?

5. Tem tremores nas mãos?

6. Sente-se nervoso(a), tenso(a) ou preocupado(a)?

7. Tem má digestão?

8. Tem dificuldade de pensar com clareza?

9. Tem se sentido triste ultimamente?

10. Tem chorado mais do que de costume?

11. Encontra dificuldades para realizar com satisfação suas atividades diárias?

12. Tem dificuldades para tomar decisões?

13. Tem dificuldades no serviço (seu trabalho é penoso, lhe causa sofrimento)?

14. É incapaz de desempenhar um papel útil em sua vida?

15. Tem perdido o interesse pelas coisas?

16. Você se sente uma pessoa inútil, sem préstimo?

17. Tem tido a idéia de acabar com a vida?

19. Tem sensações desagradáveis no estomâgo? $\quad 20,0$

20. Você se cansa com facilidade? $\quad 48,0$

21. Sente que alguém quer lhe fazer mal? 20,0

22. Você é alguém muito mais importante do que a maioria das pessoas pensa? 0

23. Ouve vozes que não sabe de onde vêm, ou que outras pessoas não podem ouvir? $\quad 16,0$ 
Tabela 2. Distribuição de pacientes com sintomatologia depressiva, ideação suicida e paranóide, alucinações auditivas e morbidade psiquiátrica significativa quanto a idade e sexo e MMSE.

\begin{tabular}{|c|c|c|c|c|c|c|c|c|c|c|c|}
\hline & & \multicolumn{4}{|c|}{ Idade } & \multicolumn{3}{|c|}{ Sexo feminino } & \multicolumn{3}{|c|}{ MMSE } \\
\hline & & $\mathrm{n}(\%)$ & média & $\mathrm{t}$ & $\mathrm{p}$ & $\%$ & $\chi^{2}$ & $\mathrm{p}$ & média & $\mathrm{t}$ & $\mathrm{p}$ \\
\hline \multirow[t]{2}{*}{ Depressão } & não & $23(30,7)$ & 75,35 & \multirow[t]{2}{*}{1,49} & \multirow[t]{2}{*}{0,141} & 52,2 & \multirow[t]{2}{*}{0,85} & \multirow[t]{2}{*}{0,358} & 18,14 & \multirow[t]{2}{*}{1,18} & \multirow[t]{2}{*}{0,240} \\
\hline & $\operatorname{sim}$ & $52(69,3)$ & 72,10 & & & 63,5 & & & 15,94 & & \\
\hline \multirow{2}{*}{$\begin{array}{l}\text { Ideação } \\
\text { suicida }\end{array}$} & não & $67(89,3)$ & 73,82 & \multirow[t]{2}{*}{2,12} & \multirow[t]{2}{*}{0,037} & 61,2 & \multirow[t]{2}{*}{0,37} & \multirow[t]{2}{*}{0,541} & 16,30 & \multirow[t]{2}{*}{$-1,16$} & \multirow[t]{2}{*}{0,250} \\
\hline & $\operatorname{sim}$ & $8(10,7)$ & 67,00 & & & 50,0 & & & 19,83 & & \\
\hline \multirow{2}{*}{$\begin{array}{l}\text { Sintomas } \\
\text { paranóides }\end{array}$} & não & $60(80,0)$ & 73,82 & \multirow[t]{2}{*}{1,43} & \multirow[t]{2}{*}{0,156} & 58,3 & \multirow[t]{2}{*}{0,35} & \multirow[t]{2}{*}{0,556} & 16,53 & \multirow[t]{2}{*}{$-0,18$} & \multirow[t]{2}{*}{0,815} \\
\hline & $\operatorname{sim}$ & $15(20,0)$ & 70,20 & & & 66,7 & & & 16,92 & & \\
\hline \multirow[t]{2}{*}{ Alucinações } & não & $63(84,0)$ & 72,44 & \multirow[t]{2}{*}{$-1,47$} & \multirow[t]{2}{*}{0,144} & 58,7 & \multirow[t]{2}{*}{0,26} & \multirow[t]{2}{*}{0,607} & 17,38 & \multirow[t]{2}{*}{2,31} & \multirow[t]{2}{*}{0,024} \\
\hline & $\operatorname{sim}$ & $12(16,0)$ & 76,50 & & & 66,7 & & & 11,90 & & \\
\hline \multirow[t]{2}{*}{$\mathrm{SRQ} \geq 8$} & não & $41(54,7)$ & 75,46 & \multirow[t]{2}{*}{2,66} & \multirow[t]{2}{*}{0,009} & 58,5 & \multirow[t]{2}{*}{0,08} & \multirow[t]{2}{*}{0,776} & 17,18 & \multirow[t]{2}{*}{0,758} & 0,451 \\
\hline & $\operatorname{sim}$ & $34(45,3)$ & 70,23 & & & 61,8 & & & 15,87 & & \\
\hline
\end{tabular}

MMSE=Mini-Exame do Estado Mental $^{9}$

Sintomas relacionados a tensão e depressão eram altamente prevalentes, sendo que $45,3 \%$ dos pacientes avaliados obtiveram escores iguais ou maiores a 8 no SRQ-20.

Dois dos sintomas característicos de depressão — tristeza (SRQ-9) e perda de interesse pelas coisas (SRQ-15) - foram agrupados com o objetivo de investigar a presença de sintomatologia depressiva entre os pacientes da amostra. Cinquenta e dois (69,3\%) pacientes relataram a presença de pelo menos um desses sintomas. A Tabela 2 descreve a distribuição daqueles com sintomas depressivos, ideação suicida e paranóide, alucinações auditivas e morbidade psiquiátrica significativa $($ SRQ-20 $\geq 8)$ quanto a idade e sexo. Não se observou diferença entre os grupos quanto à distribuição por sexo. Idosos com ideação suicida e morbidade psiquiátrica importante eram significativamente mais jovens que os demais (Tabela 2). Todos os pacientes que relataram ideação suicida apresentavam sintomas característicos de depressão: tristeza ou perda de interesse. Além disso, pacientes que descreveram a presença de alucinações auditivas apresentavam escores significativamente menores no MMSE (Tabela 2).

\section{DISCUSSÃO}

Os resultados deste estudo indicam que praticamente metade dos pacientes que recebem o diagnóstico de demência em um serviço ambulatorial especializado apresentam morbidade psiquiátrica significativa. A prevalência é ainda maior se sintomas (ou grupos de sintomas) são avaliados de forma isolada. Por exemplo, nervosismo, tensão ou preocupação estavam presentes em $69 \%$ da amostra. Estes resultados indicam uma taxa de morbidade psiquiátrica semelhante àquela descrita em outros países para pacientes com demência atendidos em clínicas especializadas ou vivendo na comunidade ${ }^{2,13}$.

Alguns sintomas psiquiátricos eram particularmente prevalentes entre os pacientes avaliados. Indecisão e dificuldade para pensar com clareza, que estavam presentes em praticamente $2 / 3$ da amostra, podem ser considerados uma manifestação clínica direta do processo demencial. Nesse caso, esses sintomas seriam decorrentes das dificuldades cognitivas características da demência. Nervosismo e depressão também foram relatados por mais de $2 / 3$ dos pacientes, sugerindo que 
esses sintomas podem ser importante fonte de morbidade para a maioria dos pacientes avaliados em serviços ambulatoriais especializados. Burns e col. ${ }^{14}$ descreveram que $63 \%$ dos 108 pacientes com doença de Alzheimer (DA) vivendo na comunidade que foram entrevistados com o Geriatric Mental State Schedule relataram a presença de sintomas depressivos. Esses resultados contrastavam com a prevalência de depressão de $23 \%$ observada pelo entrevistador e de $43 \%$ descrita pelos cuidadores ${ }^{14}$. Conclui-se, portanto, que a prevalência de depressão entre pacientes com demência varia de acordo com o método utilizado para detecção dos sintomas - pacientes relatam uma prevalência mais elevada do que cuidadores e entrevistadores treinados em avaliação dos estado mental. Além disso, Burns e col. ${ }^{14}$ observaram que os indivíduos com DA que se queixavam de depressão apresentavam escores mais elevados no MMSE do que pacientes não deprimidos. Este achado não pôde ser confirmado entre os pacientes de nossa amostra. Uma das possiveis explicações para essa discrepância pode ser a diferença quanto à origem dos pacientes (comunidade versus serviço ambulatorial especializado). Além disso, os pacientes avaliados na Santa Casa eram menos comprometidos do ponto de vista cognitivo do que aqueles estudados por Burns e seus colegas (MMSE=13,4 e 9,2 para pacientes deprimidos e não deprimidos respectivamente). Vale ressaltar que, assim como nós, outros estudos ${ }^{15}$ também não observaram associação significativa entre a gravidade do quadro demencial e a presença de sintomatologia depressiva.

A presença de sintomatologia depressiva entre pacientes demenciados é, por vezes, interpretada como uma reação vivencial natural do paciente à percepção de que suas habilidades intelectuais estão deteriorando. Por outro lado, há evidências de que fatores biológicos podem estar envolvidos na gênese desses sintomas. Förstl e col. ${ }^{16}$ estudaram as alterações neuropatológicas de 52 pacientes com DA que evoluíram para óbito. Pacientes com história pregressa de depressão durante o curso da DA apresentavam redução significativa no número de neurônios no locus coeruleus e preservação relativa dos neurônios no núcleo basalis de Meynert e substância negra. Esses achados sugerem que a perda desproporcionalmente grande de neurônios noradrenérgicos pode representar um substrato orgânico importante da depressão em DA. Além disso, vale lembrar que doença cerebrovascular, a qual é comumente observada em pacientes com demência vascular, está intimamente associada à presença de sintomas depressivos em idosos ${ }^{17}$.

Ideação suicida foi relatada por $11 \%$ dos pacientes da amostra. Todos os pacientes que relataram o desejo de acabar com suas vidas apresentavam sintomatologia depressiva significativa e, além disso, eram mais jovens que aqueles sem esse tipo de idéia. Esses resultados sugerem que pacientes jovens com diagnóstico de demência e presença de sintomatologia depressiva devem ser cuidadosamente avaliados quanto à presença de ideação suicida. Nesses casos, a depressão deve ser adequadamente tratada e a evolução do quadro depressivo monitorizada de forma detalhada. Em estudo recente, Lawrence e col. ${ }^{18}$ investigaram a associação entre o diagnóstico de transtorno mental e suicídio. Dois porcento dos 181 casos de suicídio registrados para indivíduos com mais de 65 anos de idade entre 1980 e 1995 no estado de 'Western Australia' ocorreram em pacientes com demência. Eles observaram, ainda, que 6\% dos 691 casos de tentativa de suicídio haviam recebido o diagnóstico de demência. Esses dados indicam que atos de auto-agressão não são incomuns entre pacientes demenciados.

Ideação paranóide foi relatada por $20 \%$ dos pacientes da amostra. Neste estudo esse sintoma foi avaliado de forma simples através de uma única pergunta: 'Sente que alguém quer lhe fazer mal?'. A limitação inerente a uma avaliação tão simplificada é evidente. No entanto, Migliorelli e col. ${ }^{19}$ relataram a mesma frequência de ideação delirante entre os 103 pacientes com DA atendidos em um serviço ambulatorial especializado em Buenos Aires, Argentina. Taxas semelhantes foram descritas por outros pesquisadores ${ }^{20}$ entre indivíduos portadores de DA vivendo na comunidade. Além disso, não observamos diferença significativa entre pacientes com e sem ideação paranóide quanto à idade, sexo, ou escores no MMSE. Esses achados estão de acordo com os resultados observados em outros estudos ${ }^{19,20}$. Alguns estudos relataram que os cérebros de pacientes demenciados com delírios são mais bem preservados em medidas de tomografia computadorizada que daqueles 
pacientes sem esses sintomas ${ }^{20,21}$, o que está de acordo com os achados neuropatológicos mostrando que pacientes psicóticos têm preservação relativa no número de neurônios presentes no giro parahipocampal ${ }^{22}$. Esse achado tem sido interpretado como indicativo de que a presença de idéias complexas como delírios só é possível em um cérebro que esteja relativamente preservado ${ }^{20,21}$.

Outros sintomas psicóticos que podem ser observados entre pacientes com demência são alucinações. Alucinações visuais parecem ser mais frequentes que alucinações auditivas ${ }^{23}$, particularmente entre os pacientes portadores de demência com corpúsculos de Lewy ${ }^{24}$. O presente estudo não investigou a presença de alucinações visuais, embora $16 \%$ dos pacientes relatassem ouvir vozes. Burns e col. ${ }^{23}$ observaram que indivíduos que descreviam a presença de alucinações apresentavam deterioração mais rápida nos escores do MMSE durante seguimento de 1 ano. Esse dado é compatível com nosso achado de que pacientes com alucinações são mais comprometidos cognitivamente. Entretanto, a generalização desse resultado fica comprometida pela maneira simples como a presença de alucinações foi avaliada neste estudo.

Este estudo apresenta algumas limitações metodológicas. Dezessete porcento dos pacientes com diagnóstico estabelecido de demência precisaram ser excluídos devido à pouca confiabilidade no relato dos sintomas. É possível que esses pacientes apresentassem quadro demencial mais grave ou que estivessem particularmente perturbados mentalmente. Se esse for de fato o caso é possível que a inclusão deles tivesse alterado a prevalência de alguns dos sintomas psiquiátricos acima relatados. Além disso, não foi possível estabelecer se os dados observados neste serviço ambulatorial são representativos do que ocorre entre os pacientes com demência vivendo na comunidade brasileira. A ausência de dados publicados sobre o assunto impossibilitou uma comparação direta de nossos resultados. Uma outra limitação deste trabalho refere-se à impossibilidade de esclarecer a causa de demência entre os pacientes do estudo - diferentes tipos de demência podem estar associados a distribuição diferente de sintomas psiquiátricos. Cabe, ainda, destacar que o instrumento utilizado para investigar a presença de morbidade psiquiátrica entre os nossos pacientes (SRQ-20) não foi, ainda, adequadamente validado para esse propósito. Escalas como o BEHAVE-AD ${ }^{25}$, 'Neuropsychiatric Inventory ${ }^{26}$ ' e a 'Manchester and Oxford University Scale for the Psychopathological Assessment of Dementia ${ }^{27}$ ' têm sido preferencialmente utilizadas com essa finalidade. No entanto, vale ressaltar que o SRQ-20 foi adequadamente validado para o rastreamento de morbidade psiquiátrica em nosso meio ${ }^{11}$, inclusive entre indivíduos idosos ${ }^{12}$.

Em resumo, este estudo demonstrou que $45 \%$ dos pacientes com o diagnóstico de demência atendidos em um serviço ambulatorial especializado da cidade de São Paulo apresentam morbidade psiquiátrica significativa. Nervosismo, tensão e depressão foram particularmente frequentes entre esses pacientes. Sintomas psicóticos como ideação delirante e alucinações auditivas também eram comuns. Os profissionais de saúde trabalhando com indivíduos portadores de demência devem estar alertas para a presença de sintomas psiquiátricos entre seus pacientes, particularmente porque há evidência de que estes podem responder de forma adequada ao tratamento com psicofármacos ${ }^{28-30}$.

Agradecimentos - Agradeço aos doutores Sérgio Tamai e Regiane Garrido pela colaboração na coleta dos dados e a Leonete Faria Arruda pelo digitamento do banco de dados para este estudo. Agradeço, ainda, aos pacientes e seus cuidadores por terem contribuído com a coleta de informações que possibilitou a realização deste estudo.

\section{REFERÊNCIAS}

1. World Health Organisation. ICD-10 Classification of mental and behavioural disorders. Geneva: World Health Organisation, 1992.

2. Yeager BF, Farnett LE, Ruzicha SA. Management of the behavioral manifestations of dementia. Arch Intern Med 1995; 155:250-560.

3. Hope T, Keene J, Fairburn CG, Jacoby R, McShane R. Natural history of behavioural changes and psychiatric symptoms in Alzheimer's disease. Br J Psychiatry 1999;174:39-44.

4. McShane R, Keene J, Fairburn C, Jacoby R, Hope T. Psychiatric symptoms in patient with dementia predict the later development of behavioural abnormalities. Psychol Med 1998;28:1119-1127. 
5. Garrido R, Almeida OP. Distúrbios do comportamento em pacientes com demência: impacto sobre a vida do cuidador. Arq Neuropsiquiatr 1999;2B:427-434.

6. Coen RF, Swanwick GRJ, O'Boyle CA, Coakley D. Behaviour disturbance and other predictors of carer burden in Alzheimer's disease. Int J Geriatr Psychiatry 1997;12:331-336.

7. Donaldson C, Tarrier N, Burns A. Determinants of carer stress in Alzheimer's disease. Int J Geriatr Psychiatry 1998;13:248256.

8. Almeida OP, Garrido R, Tamai S. Unidade para idosos (UNID) do Departamento de Saúde Mental da Santa Casa de São Paulo: características clínicas de pacientes atendidos em nível ambulatorial. J Bras Psiquiatria 1998;47:291-296.

9. Almeida OP. Mini Exame do Estado Mental e o diagnóstico de demência no Brasil. Arq Neuropsiquiatr 1998;56:605-612.

10. Harding TW, Climent C, Diop M, et al. The WHO collaborative study on strategies for extending mental health care II: the development of new research methods. Am J Psychiatry 1983;140:1474-1480.

11. Mari JJ, Williams P. A validity study of a psychiatric screening questionnaire (SRQ-20) in primary care in the city of São Paulo. Br J Psychiatry 1986;148:23-26.

12. Almeida OP, Forlenza OV, Lima NKC, et al. Psychiatric morbidity among the elderly in a primary care setting: report from a survey in São Paulo, Brazil. Int J Geriatr Psychiatry 1997;12:728-736.

13. Burns A, Levy R Clinical diversity in late onset Alzheimer's disease, London: Oxford Univ Press, 1992.

14. Burns A, Jacoby R, Levy R. Psychiatric phenomena in Alzheimer's disease: III. Disorders of mood. Br J Psychiatry 1990;157:81-86.

15. Cummings J, Miller B, Hill MA, et al. Neuropsychiatric aspects of multi-infarct dementia and dementia of the Alzheimer type. Arch Neurol 1987;44:389-393.

16. Förstl H, Burns A, Luthert P, et al. Clinical and neuropathological correlates of depression in Alzheimer's disease. Psychol Med 1992;22:877-884.

17. Alexopoulos GS, Meyers BS, Young RC, et al. 'Vascular depression' hypothesis. Arch Gen Psychiatry 1997;54:915-922.

18. Lawrence D, Almeida OP, Hulse G, et al. Depression and attempted suicide in later life: association with mental illness. (submitted).

19. Migliorelli R, Tesón PA, Sabe L, Leiguarda R, Starkstein SE. Neuropsychiatric and neuropsychological correlates of delusions in Alzheimer's disease. Psychol Med 1995;25:505-513.

20. Burns A, Jacoby R, Levy R. Psychiatric phenomena in Alzheimer's disease: I. Disorders of thought content. Br J Psychiatry 1990;157:72-76.

21. Jacoby R, Levy R. Computed tomography in the elderly: 2. Senile dementia: diagnosis and functional impairment. Br J Psychiatry 1980;136:256-269.

22. Förstl H, Burns A, Levy R, Cairns N. Neuropathological correlates of psychotic phenomena in confirmed Alzheimer's disease. Br J Psychiatry 1994;165:53-59.

23. Burns A, Jacoby R, Levy R. Psychiatric phenomena in Alzheimer's disease: II. Disorders of perception. Br J Psychiatry 1990;157:76-81.

24. Ballard C, McKeith I, Harrison R, et al. A detailed phenomenological comparison of complex visual hallucinations in dementia with Lewy bodies and Alzheimer's disease. Int Psychogeriatr 1997;9:381-388.

25. Reisberg B, Auer SR, Monteiro IM. Behavioral pathology in Alzheimer's disease (BEHAVE-AD) rating scales. Int Psychogeriatr 1996;8(Suppl.3):301-308.

26. Cummings JL, Mega M, Gray K, et al. The neuropsychiatric inventory: comprehensive assessment of psychopathology in dementia. Neurology 1994;44:2308-2314.

27. Allen NHP, Gordon S, Hope T, Burns A. Manchester and Oxford University Scale for the psychopathological assessment of dementia. Int Psychogeriatr 1997;9(Suppl.1):137-142.

28. Katona CL, Hunter BN, Bray J. A double-blind comparison of the efficacy and safety of paroxetine and imipramine in the treatment of depression with dementia. Int J Geriatr Psychiatry 1998;13:100-108.

29. Devenand DP, Marder K, Michaels KS, et al. A randomized, placebo-controlled dose-comparison trial of haloperidol for psychosis and disruptive behaviors in Alzheimer's disease. Am J Psychiatry 1998;155:1512-1520.

30. Katz IR, Jeste DV, Mintzer JE, et al. Comparison of risperidone and placebo for psychosis and behavioral disturbances associated with dementia: a randomized, double-blind trial. J Clin Psychiatry 1999;60:107-115. 